\title{
Otimização dos parâmetros operacionais de eletrocoagulação aplicada à recuperação de efluentes de lavagem de veículos
}

\author{
Optimization of electrocoagulation operating \\ parameters applied to the recovery vehicle washing wastewater
}

\section{Angelita Aparecida Ribeiro da Silva', Carlos Alberto Paulinetti da Camara², Ilza Lobo ${ }^{3}$, leda Spacino Scarmínio ${ }^{4}$, João Carlos Alves ${ }^{5}$}

口-

\begin{abstract}
RESUMO
No Brasil, cerca de 32.700 postos de lavagem de veículos consomem 3,7 milhões de $\mathrm{m}^{3} / \mathrm{mês}^{-1}$, média de $60 \mathrm{~L}$ por veículo. Esse consumo poderia ser minimizado, se o efluente fosse devidamente tratado e reutilizado. Como diversos tipos de efluentes têm sido eficientemente tratados por técnicas eletroquímicas, como a eletrocoagulação (EC) e a eletroflotação (EF), o objetivo principal deste estudo foi desenvolver um sistema de tratamento de efluentes de lavagem de carros por EC, usando eletrodos de alumínio. Parâmetros como pH inicial, corrente elétrica aplicada e tempo de eletrólise foram otimizados por meio de análises físico-químicas e estatísticas. Em sua condição ótima, pH 5, corrente elétrica de 3,5 A e tempo de 20 minutos, o sistema conseguiu reduzir em $73 \%$ a demanda química de oxigênio (DQO), 58\% de surfactantes e no mínimo 90\% a turbidez e os sólidos suspensos totais (SST). Além da simples implantação e operação, a técnica mostrou-se eficiente na recuperação desse tipo de efluente, podendo ser utilizada em sistemas de reúso de áqua de lavagem de veículos.
\end{abstract}

Palavras-chave: reúso de água; eletrodos de alumínio; remoção de DQO.

\begin{abstract}
In Brazil, about 32,700 vehicle washing stations consume 3,7 million $\mathrm{m}^{3}$ per month', average of $60 \mathrm{~L}$ per vehicle. Consumption could be minimized if the effluent was properly treated and reused. As different types of effluents have been effectively treated by electrochemical techniques, such as electrocoagulation (EC) and electrocoagulation (EF), the main objective of this study was to develop a treatment system of carwash wastewater, by EC using electrodes aluminum. Parameters like the initial pH, applied electric current and electrolysis time were optimized by means of physical-chemical analysis and statistics. In its optimum condition, $\mathrm{pH}$ 5, electrical current of 3,5 A and time of 20 minutes, the system has managed to reduce by $73 \%$ the chemical oxygen demand (COD), 58\% of surfactants and a minimum of $90 \%$ turbidity and total suspended solids (TSS). Besides the simple installation and operation, the technique was efficient in the recovery of this type of effluent and can be used in reuse system carwash water.
\end{abstract}

Keywords: water reuse; aluminum electrodes; COD removal.

\section{INTRODUÇÃO}

Efluentes de lavagem de carro merecem destaque por conta do grande volume de água consumida. Segundo Leão et al. (2010), no Brasil, cerca de 32.700 postos de lavagem consomem 3,7 milhões de $\mathrm{m}^{3}$ por mês, o equivalente ao consumo mensal de uma cidade de 600 mil habitantes. Um estudo realizado no município de Pombal (PB) estimou que a lavagem de um único veículo consome em média $60 \mathrm{~L}$ de água (ALVES et al., 2014). Em função da toxicidade de sua composição, detergentes, solventes, óleos e graxas, metais pesados etc., o descarte indevido desse tipo de efluente preocupa (PANIZZA \& CERISOLA, 2010b).
A Lei $n .^{\circ}$ 16.160/15 obriga postos de combustíveis e lava-rápidos da capital paulista a adotar um programa de reúso de água (PREFEITURA DO MUNICÍPIO DE SÃO PAULO, 2015). No Reino Unido, se a reciclagem e reutilização não forem possíveis, descarregar o efluente de lavagem de veículos em um esgoto público é geralmente a melhor opção ambiental, uma vez que o efluente élevado para uma estação de tratamento de esgoto (SEPA, 2007). Na Alemanha e em outros países europeus, a proibição da lavagem do carro em ruas está em vigor em várias cidades (THE TELEGRAPH, 2012).

A eletrocoagulação (EC) e a eletroflotação (EF) têm atraído atenção como tratamentos de efluentes industriais, pela alta eficiência e

'Doutoranda em Química pela Universidade Estadual de Londrina (UEL) - Londrina (PR), Brasil.

²Doutor em Química pela Universidade de São Paulo (USP) - São Carlos (SP), Brasil.

3Doutora em Química Ambiental pela Universidade Federal de São Carlos (UFCAS) - São Carlos (SP), Brasil

4Pós-doutora pela Chalmers University of Technology - Gothenburg, Suécia.

${ }^{5}$ Doutor em Química pela USP - São Paulo (SP), Brasil.

Endereço para correspondência: Angelita Aparecida Ribeiro da Silva - Rodovia Celso Garcia Cid, Km 380, s/n - Campus Universitário - 86057-970 - Londrina (PR), Brasil -

E-mail: angelita@uel.br

Recebido em: 06/02/13 - Aceito em: 31/05/2016 - Reg. ABES: 111063 
por seu funcionamento simples. Vários tipos de efluentes têm sido tratados com sucesso por EC e EF: efluentes domésticos (KURT et al., 2008), efluentes de galvanoplastia (AKBAL \& CAMCÝ, 2011; ADHOUM et al., 2004), efluentes oleosos (BENSADOK et al., 2008; TIR \& MOSTEFA, 2008), efluentes têxteis (ZODI et al., 2009; ZONGO et al., 2009), efluentes de indústrias alimentícias (TCHAMANGO et al., 2010), efluentes de lavanderias (WANG; CHOU; KUO, 2009) e também efluentes de lavagem de veículos (BAZRAFSHAN et al., 2012; PANIZZA \& CERISOLA, 2010a; 2010b).

A EC é um processo eletrolítico que consiste na dissolução de anodos de sacrifício, geralmente de ferro ou alumínio, pela aplicação de corrente elétrica entre os eletrodos. $\mathrm{Na}$ dissolução, são gerados íons $\mathrm{Al}^{3+} \mathrm{ou}$ $\mathrm{Fe}^{2+} / \mathrm{Fe}^{3+}$ (anodo) e hidroxila no cátodo, formando hidróxidos metálicos, os quais agem como agentes coagulantes para os poluentes suspensos no efluente (BEHBAHANI; MOGHADDAM; ARAMI, 2011;ZODI et al., 2009).

Os complexos hidroxicatiônicos gelatinosos $\mathrm{Al}_{\mathrm{n}}(\mathrm{OH})_{3 \mathrm{n}}$ atuam na remoção de poluentes por adsorção. Durante o processo eletrolítico, podem ocorrer simultaneamente a eletrocoagulação, a eletroflotação e a eletro-oxidação. A eletroflotação é um processo no qual as bolhas de gases $\left(\mathrm{H}_{2}\right.$ e $\left.\mathrm{O}_{2}\right)$ geradas durante a eletrólise são levadas para a superfície do efluente no reator, juntamente com os materiais poluentes. Assim, é formada uma camada de espuma, contendo bolhas de gás e material flotado na superfície do líquido (KUSHWAHA; SRIVASTAVA; MALL, 2010). A eletro-oxidação pode ocorrer ou por oxidação anódica, na qual o poluente é adsorvido e oxidado na superfície do eletrodo, ou por oxidação indireta, na qual alguns agentes oxidantes são gerados eletroquimicamente, sendo responsáveis pela oxidação dos poluentes (KUSHWAHA; SRIVASTAVA; MALL, 2010).

A eficiência dos processos de EC é controlada por várias condições, como densidade de corrente, $\mathrm{pH}$, material dos eletrodos e quantidade de matéria a ser removida, bem como íons coexistentes (BENSADOK et al., 2008). A presença de íons cloreto aumenta a condutividade, inibe a formação de óxidos nos eletrodos e forma cloro ativo (cloro, ácido hipocloroso e/ou hipoclorito), que remove poluentes por oxidação (BENSADOK et al., 2008).

Além de mais rápida, a EC apresenta menor custo operacional em relação à coagulação química convencional, cerca de 3,2 vezes (MAHMUT; MURAT; MEHMET, 2007). Comparando o consumo energético entre eletrodos de ferro e alumínio utilizados em EC, verificou-se menor consumo para eletrodos de alumínio, no entanto esse tipo de material tem custo mais elevado (LACASA et al., 2013). Para minimizar custos, células solares são uma opção sustentável quando associadas à EC (GARCÍA et al., 2015).

De acordo com Kobya et al. (2006), o cálculo do consumo de energia em um reator batelada é expresso pela Equação 1.

$\mathrm{C}$ Energia $=(\mathrm{U} . \mathrm{i} . \mathrm{t}) / \mathrm{V}$
Em que:

C energia = consumo de energia em W.h. $\mathrm{m}^{-3}$;

$\mathrm{U}=$ tensão elétrica aplicada no sistema, em V;

$\mathrm{i}=$ corrente elétrica aplicada, em A;

$\mathrm{t}=$ tempo de aplicação da corrente em $\mathrm{h}$;

$\mathrm{V}=$ volume de efluente tratado $\mathrm{em}^{3}$.

A corrosão do eletrodo contribui para o custo de operação e segue a lei de Faraday, podendo ser calculada de acordo com a Equação 2:

C eletrodo $=($ M.I.t $) /($ ZFV $)$

Em que:

C eletrodo = custo do eletrodo;

$\mathrm{M}=$ massa atômica do alumínio $\left(28,98 \mathrm{~g} \mathrm{~mol}^{-1}\right)$;

$\mathrm{I}=$ corrente, em A;

$\mathrm{t}=$ tempo de reação, em $\mathrm{h}$;

$\mathrm{Z}=$ número de elétrons transferidos $(\mathrm{Z}=3)$;

$\mathrm{F}=$ constante de Faraday $\left(96487 \mathrm{C} \mathrm{mol}^{-1}\right)$;

$\mathrm{V}=$ volume do efluente a ser tratado, em $\mathrm{m}^{3}$ (GHOSH; MEDHI; PURKAIT, 2008).

O objetivo do trabalho foi investigar a eficiência da EC com eletrodos de alumínio, para remoção da demanda química de oxigênio (DQO) e turbidez, em efluentes de lavagem de veículos. Um arranjo do tipo composto central e a metodologia de superfície de resposta foram usados. Análises estatísticas indicaram a condição ótima do tratamento por EC, avaliando os efeitos dos parâmetros: $\mathrm{pH}$ inicial, densidade de corrente elétrica e tempo de eletrólise. O custo operacional, considerando os gastos de energia elétrica e material, também foi avaliado.

\section{METODOLOGIA}

\section{Coleta da amostra e caracterização}

O efluente estudado, proveniente de lavagem de carros, foi coletado em três dias diferentes em um lava-rápido de um posto de combustível localizado na cidade de Londrina, Paraná, Brasil. No local, o efluente gerado foi armazenado em três caixas separadoras de óleo e água, feitas em alvenaria e com dimensões de $100 \times 50 \times 50 \mathrm{~cm}$. Nessas caixas, antes da filtragem final em areia e da descarga na rede de esgoto, os materiais mais densos sofreram decantação e os menos densos flotação.

\section{Planejamento experimental}

O efluente coletado foi homogeneizado e submetido a diversas condições de tratamento, seguindo um planejamento experimental do tipo composto central, que combinou três variáveis (tempo de 
eletrólise, densidade de corrente e $\mathrm{pH}$ inicial), explorando diversas faixas de valores explorados e já publicados (ABOULHASSAN et al., 2006; BENSADOK et al., 2008; GÁRCIA et al., 2015), e cinco níveis -1,68, -1, $0,1,1,68$, conforme o planejamento criado pelo software Statistica 7.0. Esse tipo de planejamento permite a análise simultânea do efeito de cada variável e suas interações e determina, por exemplo, as variáveis mais significativas para a resposta desejada — como $\mathrm{pH}$, tempo de eletrólise e corrente elétrica aplicada.. A Tabela 1 apresenta a combinação entre essas variáveis.

No total foram realizados 17 experimentos, calculados seguindo a Equação 3 (MANSOUR \& KESENTINI, 2008).

$\mathrm{N}=2^{\mathrm{k}}+2 \mathrm{k}+\mathrm{n}_{0}$

Em que:

$\mathrm{N}$ = número de experimentos realizados (17);

$\mathrm{n}_{0}=$ número de repetições no centro do plano (3);

$\mathrm{k}=$ número de variáveis independentes (3).

As análises estatísticas foram realizadas com base nas taxas de remoção da DQO (Equação 4). Avaliaram-se os modelos linear, quadrático e quadrático + linear, a fim de decidir o mais adequado. Tendo os resultados do coeficiente de correlação e análise da tabela ANOVA, verificou-se que o modelo quadrático + linear foi o que mais se adequou para descrever a taxa de remoção de DQO, apresentando a melhor correlação entre os valores observados e previstos, $\left(R^{2}\right)$ de 0,96654 .

Tabela 1 - Combinação entre as variáveis: $\mathrm{pH}$, corrente elétrica e tempo, aplicadas em cada tratamento, conforme o planejamento experimental.

\begin{tabular}{|c|c|c|c|}
\hline Experimentos & $\mathrm{pHi}$ & I (A) & $\mathrm{t}(\mathrm{min})$ \\
\hline 1 & 5,00 & 1,50 & 10,0 \\
\hline 2 & 5,00 & 1,50 & 20,0 \\
\hline 3 & 5,00 & 3,50 & 10,0 \\
\hline 4 & 5,00 & 3,50 & 20,0 \\
\hline 5 & 9,00 & 1,50 & 10,0 \\
\hline 6 & 9,00 & 1,50 & 20,0 \\
\hline 7 & 9,00 & 3,50 & 10,0 \\
\hline 8 & 9,00 & 3,50 & 20,0 \\
\hline 9 & 7,00 & 2,50 & 15,0 \\
\hline 10 & 7,00 & 2,50 & 15,0 \\
\hline 11 & 7,00 & 2,50 & 15,0 \\
\hline 12 & 7,00 & 2,50 & 6,6 \\
\hline 13 & 7,00 & 2,50 & 23,4 \\
\hline 14 & 7,00 & 0,82 & 15,0 \\
\hline 15 & 7,00 & 4,18 & 15,0 \\
\hline 16 & 3,64 & 2,50 & 15,0 \\
\hline 17 & 10,36 & 2,50 & 15,0 \\
\hline
\end{tabular}

pHi: pH inicial; I: corrente; t: tempo.
Também foram avaliados os efeitos das variáveis independentes e suas interações (gráfico de Pareto) e a superfície de resposta.

\% Remoção de DQO = $\mathrm{DQO}_{\mathrm{ST}}-\mathrm{DQO}_{\mathrm{T}} / \mathrm{DQO}_{\mathrm{ST}}$

Em que:

$\mathrm{DQO}_{\mathrm{ST}}=$ valor da DQO no efluente sem tratamento;

$\mathrm{DQO}_{\mathrm{T}}=$ valor de DQO no efluente tratado.

\section{Experimentos de eletrocoagulação}

Os experimentos de EC foram conduzidos utilizando dois eletrodos de alumínio, um cátodo e um ânodo, dispostos verticalmente, acoplados a um reator (recipiente plástico) e conectados a uma fonte elétrica (FA 1030 Instrutherm DC Power Supply). O volume total do efluente tratado foi de $2.000 \mathrm{~cm}^{3}$, e os experimentos foram conduzidos à temperatura ambiente $\left(25-28^{\circ} \mathrm{C}\right)$ e agitados a $4.000 \mathrm{rpm}$ (Fisatom 752).

A condutividade específica foi ajustada a $4 \mathrm{mS} / \mathrm{cm}$ por meio de cloreto de sódio e um condutivímetro Digimed DM-31. Valores de $\mathrm{pH}$ iniciais foram ajustados em cada um dos 17 experimentos, com adição de hidróxido de sódio $0,1 \mathrm{M}$ e ácido clorídrico 0,1 Me utilização de um potenciômetro Marte MB-10. A Figura 1 ilustra o sistema experimental da EC.

\section{Análises físico-químicas}

Ao final dos experimentos, os efluentes tratados foram agitados com bastão de vidro para homogeneização e decantação de resíduos de espuma, sorvidos na superfície do reator. Em seguida foram realizadas as leituras de $\mathrm{pH}$ e condutividade finais e corrigidos todos os valores de $\mathrm{pH}$ para 7,2 utilizando hidróxido de sódio $0,1 \mathrm{M}$ ou ácido clorídrico $0,1 \mathrm{M}$, para neutralizá-los e poder descartá-los no ambiente ou reutilizá-los.

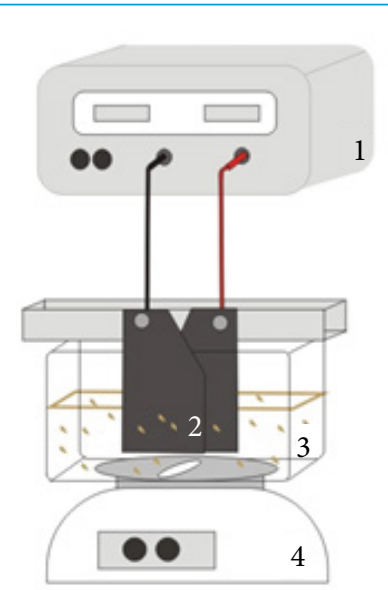

1: Fonte elétrica; 2: eletrodos de alumínio com dimensões 16,0 × 10,0 × 0,2 cm, com área efetiva de $100,0 \mathrm{~cm}^{2}$ cada face, confeccionados com chapa lisa de alumínio, com pureza de 99,5\%; 3: reator de $2 \mathrm{~L}$, com dimensões 16,0 × 12,0 × 10,5 cm;

4: agitador magnético.

Figura 1 - Diagrama esquemático da configuração experimental. 
Os experimentos foram deixados em repouso por um período de 24 horas, tempo mínimo necessário observado para que todos os tratamentos completassem o processo de coagulação.

O efluente tratado foi coletado com auxílio de uma bomba peristáltica (SV Sistemas Vitais RS 106), posicionada a $1 \mathrm{~cm}$ do lodo, e armazenado a $4^{\circ} \mathrm{C}$, para posteriores análises. O lodo residual também foi armazenado para futuras investigações e análises.

As análises realizadas neste experimento seguiram Standard methods for the examination of water and wastewater (APHA; AWWA; WEF, 1998). Para DQO, o método aplicado foi o 5220D, refluxo fechado, colorimétrico. O teor de surfactante foi determinado seguindo o Método 5540C: surfactantes aniônicos com Methylene Blue Active Substances (MBAS). Sólido suspenso total foi medido gravimetricamente, após filtração em membranas Millipore (MM) de 0,45 $\mu$ m. Mediu-se a turbidez diretamente no turbidímetro da marca Hach, faixa de medição de 0 a 1.000 NTU. Alumínio residual foi mensurado por meio de espectroscopia de emissão atômica (ICP-MS Varian 820). O lodo gerado durante o tratamento foi filtrado em papel-filtro, previamente pesado, seco ao ar por duas semanas, e seu peso determinado gravimetricamente.

Numa avaliação econômica preliminar, deviam ser considerados o consumo energético e o consumo do eletrodo (Equação 5).

$\mathrm{C}$ operacional $=\mathrm{aC}$ energia $+\mathrm{bC}$ eletrodo

Em que:

C operacional $=$ custo operacional;

Coeficiente $\mathrm{a}=$ preço da energia elétrica industrial $\left(0,38790 \mathrm{R} \$ \mathrm{k} \cdot \mathrm{w} \cdot \mathrm{h}^{-1}\right)$; $\mathrm{C}$ energia $=$ consumo de energia elétrica $\left(\mathrm{kwh} . \mathrm{m}^{-3}\right)$;

Coeficiente $b=$ preço do eletrodo de alumínio $\left(23,50918 \mathrm{R} \$ . \mathrm{kg}^{-1} \mathrm{Al}\right)$. $\mathrm{C}$ eletrodo $=$ desgaste do eletrodo $\left(\mathrm{kg} \mathrm{Al} . \mathrm{m}^{-3}\right)$;

\section{RESULTADOS E DISCUSSÃO}

O efluente coletado foi caracterizado quanto a DQO (331,26 mg.L'), sólidos suspensos totais (SST) (261,20 mg.L), surfactantes (6,50 mg.L'), turbidez (169 NTU) e condutividade $\left(616 \mu \mathrm{S} \mathrm{cm}^{-1}\right)$.

Os valores de DQO, SST, turbidez, lodo gerado e consumo energético obtidos após o tratamento por EC são apresentados na Tabela 2. A DQO final do efluente nos 17 experimentos variou de 83,91 a 237,77 $\mathrm{mg} \mathrm{O}_{2} \mathrm{~L}^{-1}$.

$\mathrm{O}$ valor final dos SST em todos os experimentos variou de 0,1 a 4,6 mg. $\mathrm{L}^{-1}$. A Companhia de Saneamento Básico do Estado de São Paulo (Sabesp) propõe que o valor ideal de SST seja de $30 \mathrm{mg} \mathrm{L}^{-1}$ para água destinada a reúso em lavagem de carros (SABESP, 2002). O uso de água que contenha elevado valor de SST pode resultar em partículas aderidas à superfície do veículo, requerendo enxágue com água limpa, para total retirada das partículas residuais. A Resolução do Conselho Nacional do Meio Ambiente (CONAMA) n. 357 não estabelece valores de referência para SST em águas naturais, de descarte ou consumo humano, no entanto são permitidos $500 \mathrm{mg} \mathrm{L}^{-1}$ de sólidos dissolvidos totais (SDT) para águas doces das classes 1, 2 e 3 e $1.000 \mathrm{mg} \mathrm{L}^{-1}$ para águas subterrâneas, classes 1 e 2 (BRASIL, 2002).

Os valores finais de turbidez variaram de 1,01 a 12,67 NTU. Segundo Hespanhol et al. (2006), águas reutilizadas em processos de lavagem de veículos devem apresentar turbidez menor que 5 NTU

Cinco dos 17 experimentos realizados, os de número 4, 8, 13, 15 e 16 , apresentaram os maiores valores de \% de remoção de DQO e turbidez após o tratamento (Tabela 3). A corrente elétrica e o tempo aplicados nos experimentos 4 e 8 foram iguais (3,5 A e 20 minutos), diferenciando somente o $\mathrm{pH}$ inicial, 5 e 9 , respectivamente. O experimento feito em menor $\mathrm{pH}$ inicial mostrou-se mais eficiente. Acredita-se que essa maior eficiência se deva à baixa solubilidade do hidróxido de alumínio [ $\left.\mathrm{Al}(\mathrm{OH})_{3}\right]$, em $\mathrm{pH}$ por volta de 4 . O hidróxido de alumínio não solubilizado atua como um agente coagulante, removendo por adsorção poluentes da água (PICHARD et al., 2000; CAÑIZARES et al., 2005). Tir e Mostefa (2008) concluíram que em pH maior que 9 os flocos de hidróxido de alumínio são menos reativos e a floculação menos efetiva, pela formação de flocos de pequenos tamanhos que compõem um depósito no ânodo e conduzem a um aumento da resistência ôhmica.

Tabela 2 - Valores médios de demanda química de oxigênio, sólidos suspensos totais, turbidez e lodo obtidos após o tratamento por eletrocoagulação, seguindo diferentes condições de $\mathrm{pH}$, intensidade de corrente e tempo.

\begin{tabular}{|c|c|c|c|c|c|c|}
\hline Experimentos & $\begin{array}{c}\text { DQO } \\
\mathrm{mg} \mathrm{O}_{2} \mathrm{~L}^{-1}\end{array}$ & \begin{tabular}{|l} 
SST \\
$m g \cdot L^{-1}$
\end{tabular} & $\begin{array}{c}\text { Turbidez } \\
\text { NTU }\end{array}$ & $\begin{array}{l}\text { Lodo } \\
\text { g. } \mathrm{L}^{-1}\end{array}$ & $\begin{array}{c}\text { C energético } \\
\text { Wh.m }\end{array}$ & $\begin{array}{c}\text { C eletrodo } \\
\text { g Al.m }^{3}\end{array}$ \\
\hline 1 & 219 & 3,40 & 6,65 & 0,56 & 712,5 & 0,1669 \\
\hline 2 & 201 & 1,80 & 2,95 & 0,76 & 1425,0 & 0,6674 \\
\hline 3 & 185 & 1,30 & 1,88 & 0,88 & 4025,0 & 0,1669 \\
\hline 4 & 84 & 1,20 & 1,01 & 2,24 & 8050,0 & 0,6674 \\
\hline 5 & 238 & 2,90 & 9,76 & 0,60 & 712,5 & 0,1669 \\
\hline 6 & 215 & 2,10 & 6,70 & 0,81 & 142,0 & 0,6674 \\
\hline 7 & 206 & 2,30 & 5,14 & 0,88 & 4025,0 & 0,1669 \\
\hline 8 & 178 & 1,40 & 1,57 & 0,90 & 8050,0 & 0,6674 \\
\hline 9 & 180 & 1,70 & 12,67 & 0,94 & 3281,3 & 0,3754 \\
\hline 10 & 180 & 1,30 & 3,68 & 0,92 & 3281,3 & 0,3754 \\
\hline 11 & 182 & 1,60 & 4,89 & 0,91 & 3281,3 & 0,3754 \\
\hline 12 & 238 & 2,50 & 6,08 & 0,49 & 1443,8 & O,0727 \\
\hline 13 & 161 & 4,60 & 1,90 & 0,80 & 5118,8 & 0,9137 \\
\hline 14 & 211 & 0,10 & 11,40 & 0,46 & 399,8 & 0,3754 \\
\hline 15 & 147 & 2,20 & 1,61 & 0,82 & 8725,8 & 0,3754 \\
\hline 16 & 161 & 1,00 & 1,30 & 0,93 & 3281,3 & 0,3754 \\
\hline 17 & 208 & 2,10 & 4,03 & 0,87 & 3281,3 & 0,3754 \\
\hline
\end{tabular}

DQO: demanda química de oxigênio; SST: sólidos suspensos totais; C energético: consumo energético; C eletrodo: consumo do eletrodo. 
A quantidade de lodo formada na EC está relacionada com a quantidade de material coagulante produzido e a remoção de SST e outros compostos presentes no efluente. As quantidades de lodo formado variaram de 0,4603 a 2,2368 $\mathrm{g} \mathrm{L}^{-1}$. Tratamentos por EC produzem de duas a três vezes menos lodo que a coagulação química convencional (MUSQUERE; ELLINGSEN; VIK, 1983). Tratamentos por coagulação química envolvem a adição de grandes quantidades de polímeros e sais de ferro e alumínio, contribuindo com o aumento do

Tabela 3 - Percentagem de remoção de turbidez, sólidos suspensos totais e demanda química de oxigênio: valor observado, previsto e o resíduo, obtidos diretamente pelo software Statistica 7.0.

\begin{tabular}{|c|c|c|c|c|c|}
\hline \multirow{2}{*}{ Experimentos } & \multirow{2}{*}{$\begin{array}{c}\text { \% de Remoção } \\
\text { Turbidez }\end{array}$} & \multirow{2}{*}{$\begin{array}{c}\text { \% Remoção } \\
\text { SST }\end{array}$} & \multicolumn{3}{|c|}{ \% Remoção de DQO } \\
\hline & & & Observado & Previsto & "Resíduos \\
\hline 1 & 95 & 99 & 34,987 & 32,339 & 2,648 \\
\hline 2 & 98 & 99 & 39,850 & 42,433 & $-2,582$ \\
\hline 3 & 98 & 100 & 45,316 & 46,749 & $-1,433$ \\
\hline 4 & 99 & 95 & 73,190 & 69,814 & 3,376 \\
\hline 5 & 92 & 99 & 28,658 & 32,304 & $-3,647$ \\
\hline 6 & 98 & 99 & 36,453 & 35,291 & 1,162 \\
\hline 7 & 96 & 99 & 36,680 & 34,368 & 2,312 \\
\hline 8 & 99 & 99 & 47,407 & 50,326 & $-2,919$ \\
\hline 9 & 90 & 99 & 45,146 & 44,827 & 0,318 \\
\hline 10 & 97 & 100 & 45,521 & 44,827 & 0,693 \\
\hline 11 & 96 & 99 & 43,748 & 44,827 & $-1,079$ \\
\hline 12 & 95 & 99 & 29,180 & 29,240 & $-0,059$ \\
\hline 13 & 98 & 98 & 51,566 & 51,123 & 0,443 \\
\hline 14 & 91 & 100 & 35,101 & 33,792 & 1,309 \\
\hline 15 & 99 & 99 & 57,600 & 58,525 & $-0,926$ \\
\hline 16 & 99 & 100 & 49,725 & 51,051 & $-1,326$ \\
\hline 17 & 97 & 99 & 36,362 & 34,652 & 1,710 \\
\hline
\end{tabular}

DQO: demanda química de oxigênio; SST: sólidos suspensos totais; *Resíduos: erros volume do lodo gerado (MOHAMMAD \& MUTTUCUMARU, 2009). Em 2007 a Agência de proteção ambiental dos Estados Unidos (EPA) declarou que todo lodo contendo alumínio proveniente de efluentes industriais deve ter como disposição final a incineração, a reciclagem ou o reprocessamento para separação do alumínio e posterior aplicação da biorremediação (EPA, 2016).. No Brasil, as resoluções de 2002 e 2005 do Conselho Nacional do Meio Ambiente (CONAMA) não listam o alumínio como uma substância inorgânica potencialmente tóxica; entretanto, resíduos oleosos do sistema separador água e óleo enquadram-se na classe 1 por serem considerados perigosos, devendo ser vetados na agricultura e encaminhados a aterros sanitários licenciados (BRASIL, 2002; 2011).

O consumo energético variou entre 399,8 e 8725,8 wh.m ${ }^{-3}$. A energia necessária para desenvolver o processo de EC é altamente dependente da densidade de corrente aplicada.

O teor de surfactante, determinado apenas no experimento 4 ( $\mathrm{pH} 5$; 3,5 A; 20 minutos), caiu de 6,72 no efluente bruto para 2,84 mg MBAS.L $\mathrm{L}^{-1}$. A remoção de $58 \%$ explica-se por mecanismos sortivos do surfactante a complexos hidroxicatiônicos gelatinosos formados durante a EC.

\section{Remoção de demanda química de oxigênio, sólidos suspensos totais e turbidez}

Como a DQO (Tabela 3) foi a análise que apresentou resultados com as maiores variações em suas taxas de remoção, entre 28 e 74\%, os resultados foram utilizados na análise estatística. As taxas de remoção de SST e de turbidez não variaram muito, permanecendo todas acima de $90 \%$.

O Gráfico 1 apresenta o gráfico de Pareto para avaliar os efeitos de todas as variáveis estudadas e suas interações para os modelos linear (L) e quadrático (Q), considerando a eficiência da \% de remoção da DQO. No eixo Y, têm-se as variáveis independentes e suas interações. No eixo X se tem o valor absoluto do efeito estimado, calculado pela razão entre os efeitos estimados e seus respectivos desvios padrões. Todos os valores que aparecem no gráfico de Pareto situados à direita do valor $(\mathrm{p}=0,05)$ são de elevada significância estatística.

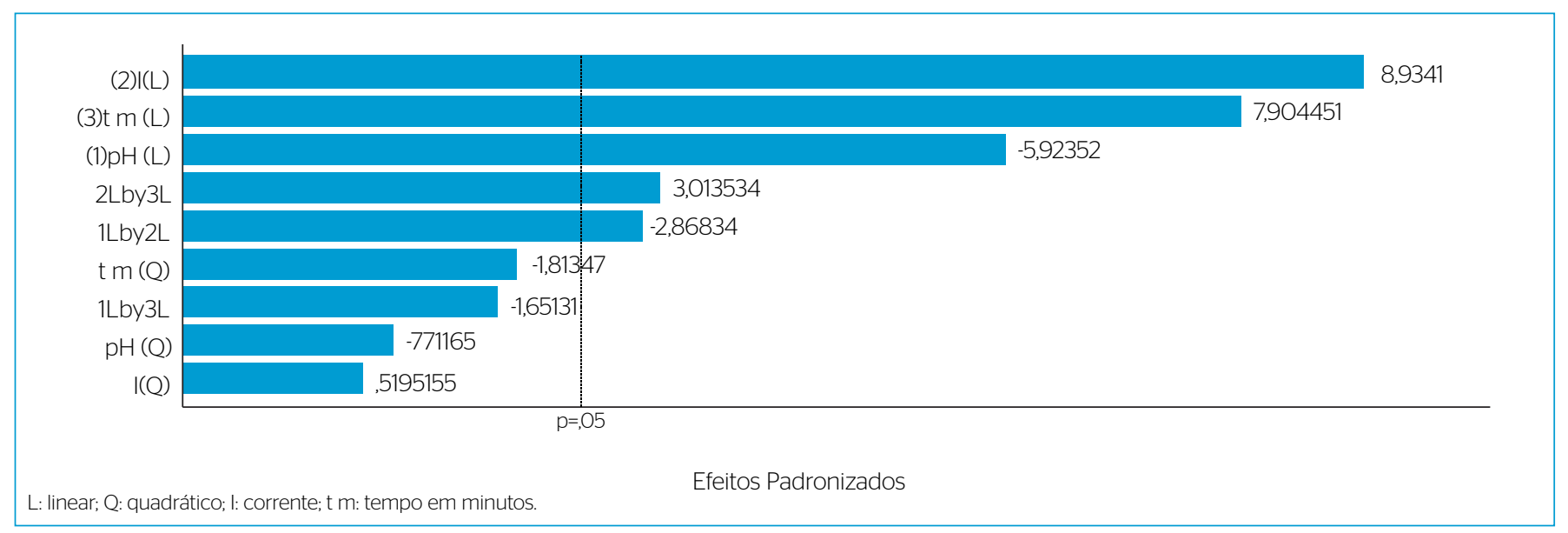

Gráfico 1 - Gráfico de Pareto para visualização dos efeitos das variáveis e de suas combinações na \% de remoção de demanda química de oxigênio. 
Observou-se que o efeito da variável corrente $(2 \mathrm{~L})$, seguida pela variável tempo $(3 \mathrm{~L})$, e a interação entre ambas (2 Lx3 L) apresentaram, respectivamente, maior significância estatística. A variável pH (1 L) demonstrou influência contrária em termos estatísticos, ou seja, à medida que se aumentaram os valores de $\mathrm{pH}$ houve decréscimo na \% de remoção de DQO. O mesmo efeito constatou-se na interação entre corrente e $\mathrm{pH}(1 \mathrm{~L} \times 2 \mathrm{~L})$. As demais interações, à esquerda do valor $(\mathrm{p}=0,05)$, não apontaram significância estatística.

$\mathrm{O}$ teste $\mathrm{F}$ foi utilizado para investigar se o modelo proposto indicava evidência estatística suficiente da relação dependente entre as variáveis de entrada e a resposta em \% de remoção de DQO (SANTOS; ALSINA; SILVA, 2007). O valor do teste F calculado deve ser maior que o valor do teste $\mathrm{F}$ tabelado para que o modelo seja significativo, ou seja, válido estatisticamente. $\mathrm{O}$ valor obtido para $\mathrm{F}_{\text {cal }}$ foi de 22,473, e viu-se que esse valor foi maior que $\mathrm{F}_{\mathrm{Tab}}=(9 ; 7 ; 0,05)=3,68$. Isto é, a regressão obtida ajustou os pontos experimentais de forma satisfatória, validando o modelo para $\mathrm{o}$ intervalo de confiança de 95\%. A Equação 6 consiste numa relação empírica expressa por uma equação polinomial de segunda ordem, em termos de interação, montada entre os resultados experimentais obtidos, com base no planejamento composto central experimental e as variáveis de entrada.

\% Remoção DQO $=-17,82+6,08^{\star} \mathrm{I}+0,47^{\star} \mathrm{I}^{\wedge} 2+2,90^{\star} \mathrm{t}-$ $0,07^{\star} \mathrm{t} \wedge 2-1,54^{\star} \mathrm{pH}^{\star} \mathrm{I}-0,181^{\star} \mathrm{pH}^{\star} \mathrm{t}+0,65^{\star} \mathrm{I}^{\star} \mathrm{t}+28,29$

Em que:

$\mathrm{t}=$ tempo do tratamento, em minutos;

$\mathrm{I}=$ corrente aplicada (A) em um determinado valor de $\mathrm{pH}$.

O valor $\mathrm{p}$ associado foi usado para estimar se F era grande o suficiente para indicar significância estatística. Valores p, observados na Tabela 4, inferiores a 0,05 mostraram que o modelo era estatisticamente significativo (KUMAR et al., 2009).
O gráfico da superfície de resposta prevista para \% de remoção de DQO baseado num modelo de segunda ordem é apresentado na Figura 3. Observou-se que a relação entre a resposta (\% remoção de DQO) e as duas variáveis, tempo e corrente, era linear. Comparando-se os três valores de $\mathrm{pH}, 5,7$ e 9, verificou-se que o pH 5 apresentou a melhor resposta do tratamento para \% de remoção de DQO, seguido pelos valores de $\mathrm{pH} 7$ e 9 . Em baixos valores de $\mathrm{pH}$, os prótons na solução foram reduzidos a $\mathrm{H}_{2}$, e então a proporção de íons hidroxila produzidos foi menor, assim como, consequentemente, a remoção de DQO (MODIRSHAHLA; BEHNAJADY; KOOSHAIIAN, 2007).

A análise gráfica de superfície de resposta para os três valores de $\mathrm{pH}(5,7$ e 9$)$ demonstrou que a resposta diminuiu conforme se aumentou o pH. O pH inicial não é um dos fatores mais importantes que afetam o desempenho dos processos eletroquímicos, mas, em $\mathrm{pH}$ alcalino, a superfície oxidada apresentou cargas negativas que tendiam a

Tabela 4 - Análise de variância (ANOVA) para a resposta \% remoção de demanda química de oxigênio (intervalo de confiança de 95\%).

\begin{tabular}{l|c|c|c|c|c}
\hline Fatores & $\begin{array}{c}\text { Soma } \\
\text { quadrática }\end{array}$ & $\begin{array}{c}\text { Graus de } \\
\text { liberdade }\end{array}$ & $\begin{array}{c}\text { Média } \\
\text { quadrática }\end{array}$ & $F$ & $p$ \\
\hline$(1) \mathrm{pH}(\mathrm{L})$ & 325,030 & 1 & 325,0340 & 35,08806 & 0,000585 \\
\hline $\mathrm{pH}(\mathrm{Q})$ & 5,509 & 1 & 5,5088 & 0,59470 & 0,465838 \\
\hline$(2) \mathrm{I}(\mathrm{L})$ & 739,390 & 1 & 739,3900 & 79,81948 & 0,000045 \\
\hline $\mathrm{I}(\mathrm{Q})$ & 2,500 & 1 & 2,5001 & 0,26990 & 0,619419 \\
\hline$(3) \mathrm{t} \min (\mathrm{L})$ & 578,773 & 1 & 578,7728 & $\mathbf{6 2 , 4 8 0 3 5}$ & 0,000098 \\
\hline $\mathrm{t}$ min (Q) & 30,464 & 1 & 30,4638 & 3,28866 & 0,112641 \\
\hline $1 \mathrm{~L} \times 2 \mathrm{~L}$ & 76,212 & 1 & 76,2122 & 8,22735 & 0,024049 \\
\hline $1 \mathrm{~L} \times 3 \mathrm{~L}$ & 25,259 & 1 & 25,2592 & 2,27681 & 0,142659 \\
\hline $2 \mathrm{~L} \times 3 \mathrm{~L}$ & 84,123 & 1 & 84,1234 & 9,08139 & 0,019564 \\
\hline Resíduos & 64,843 & 7 & 9,2633 & & \\
\hline Total SS & $1.937,779$ & 16 & & & \\
\hline
\end{tabular}

Total SS: soma total dos quadrados (do inglês Sums of Squares).

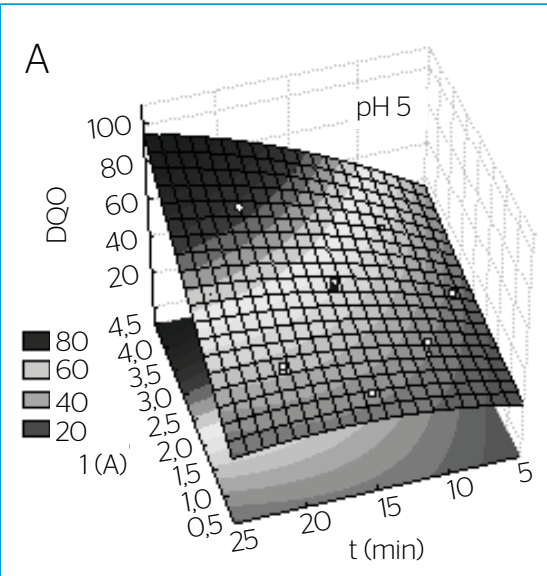

DQO: demanda química de oxigênio; l: corrente; t: tempo

Figura 3 - Superfície de resposta não linear para \% de remoção de demanda química de oxigênio em diferentes valores de pH: (A) pH 5; (B) pH 7; (C) pH 9.

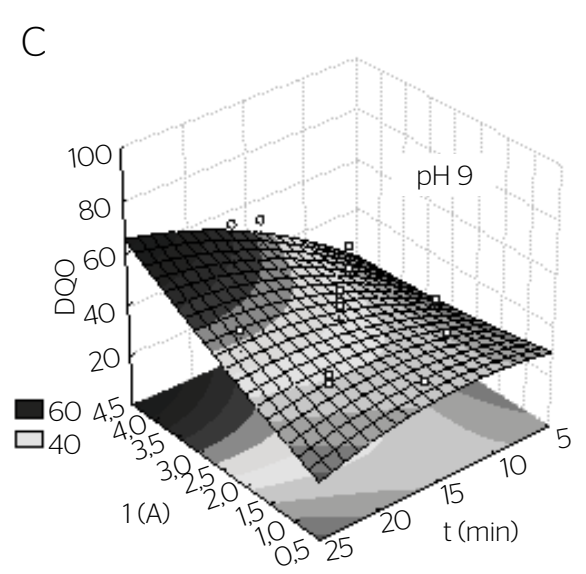

C

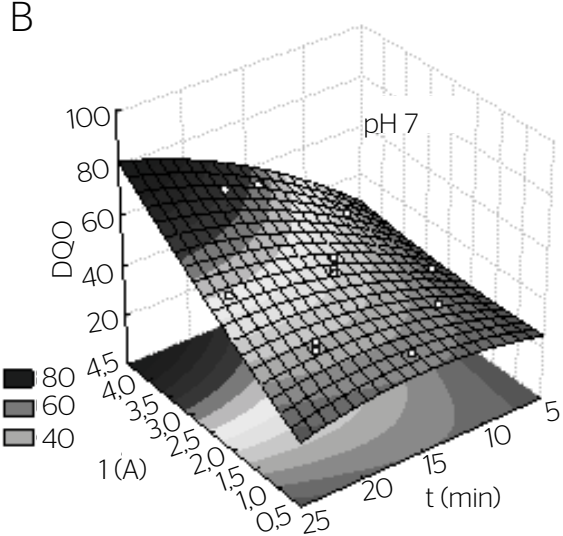


repelir os contaminantes em solução (BEHBAHANI; MOGHADDAM; ARAMI, 2011).

Após o tratamento, o experimento 4, considerado o mais eficiente estatisticamente, apresentou teor de alumínio residual igual a 3,73 \pm 0,34 mg.L . Embora o alumínio apresente elevada toxicidade, o CONAMA não exige limite máximo permitido para descarte de efluentes contendo alumínio residual. De acordo com a NBR 13.969:1997, os limites permitidos para o reúso de águas provenientes de efluentes de lavagem de carros, classificadas como classe 1, por requerem o contato direto do usuário com a água, são: turbi$\operatorname{dez}<5$, coliformes fecais $<200 \mathrm{NMP} 100 \mathrm{~mL}^{-1}$, sólidos dissolvidos totais $<200 \mathrm{mg} \mathrm{L}^{-1}, \mathrm{pH}$ entre 6,0 e 8,0 , e cloro residual entre $0,5 \mathrm{e}$ $1,5 \mathrm{mg} \mathrm{L}^{-1}$ (AMBIENTE BRASIL, 2016). O tratamento 4, avaliado como o mais eficiente, indicou $1,20 \mathrm{mg} \mathrm{L}^{-1}$ de SST, 1,20 NTU de turbidez e pH 7,2, valores que se enquadram nos limites permitidos para reúso de água.

\section{CONCLUSÃO}

Os resultados obtidos neste trabalho demonstraram que as taxas de remoção de DQO aumentaram com a elevação das taxas de densidade de corrente, tempo de eletrólise e a interação entre eles. $\mathrm{O}$ pH possui influência negativa. Ou seja, à medida que se aumentaram os valores de $\mathrm{pH}$, decresceu a \% de remoção de DQO. Conforme a análise de variância, o modelo que melhor se adequou ao experimento foi o quadrático + linear, apresentando $\mathrm{R}^{2}$ de 0,96654 . Durante a otimização do processo, foram obtidas taxas de remoção máxima de 73\% de DQO, 92\% de turbidez, 99\% de SST, 58\% de remoção de surfactantes em $\mathrm{pH}$ inicial 5, corrente 3,5 A e tempo de 20 minutos. Uma grande vantagem da eletrocoagulação em relação a outros tratamentos é a pequena quantidade de lodo gerada - as taxas variaram de 0,4603 a 2,2368 $\mathrm{g} \mathrm{L}^{-1}$. Ao considerar os valores $1,20 \mathrm{mg} \mathrm{L}^{-1}$ de SST, 1,20 NTU de turbidez e pH 7,2, obtidos no melhor tratamento, essa água pode ser reutilizada.

\section{REFERÊNCIAS}

Aboulhassan, M.A.; Souabi, S.; Yaacoubi, A.; Baudu, M. (2006) Removal of surfactant from industrial wastewaters by coagulation flocculation process. International Journal of Environmental Science and Technology, v. 3, n. 4, p. 327-332.

ADHOUM, N:; MONSER, L; BELLAKHAL, N:; BELGAIED, J.E. (2004) Treatment of electroplating wastewater containing $\mathrm{Cu}^{2+}, \mathrm{Zn}^{2+}$ and $\mathrm{Cr}(\mathrm{VI})$ by electrocoagulation. Journal of Hazardous Materials, v. 112, n. 3, p. 207-213.

AKBAL, F.; CAMCÝ, S. (2011) Copper, chromium and nickel removal from metal plating wastewater by electrocoagulation. Desalination, v. 269, n. 1, p. 214-222.

ALVES, M.F.A.; MEDEIROS, R.M.; GALVÃO, F.P.S.; SOUSA, J.A.; MELO, F.J.S.; SILVA, M.M.M.; ALVES, F.A. (2014) Cálculo estimado do volume gasto de água em dois postos de lavagem de veículos em Pombal - PB. Intesa, v. 8, n. 1, p. 10-14.

AMBIENTE BRASIL. Legislação e Normatização do Reúso da Água. Disponível em: <http://ambientes.ambientebrasil.com.br/agua/ uso_e_reúso_da_agua/legislacao_e_normatizacao_do_reúso_da_ agua.html>. Acesso em: 10 abr. 2016.

AMERICAN PUBLIC HEALTH ASSOCIATION - APHA; AMERICAN WATER WORKS ASSOCIATION - AWWA; WATER ENVIRONMENT FEDERATION - WEF. (1998) Standard methods for the examination of water and wastewater. 20th ed. New York: APHA/AWWA/WEF.

BAZRAFSHAN, E.; KORD MOSTAFAPOUR, F.; FARZADKIA, M.; OWNAGH, K.A.; MAHVI, A.H. (2012) Slaughterhouse Wastewater Treatment by Combined Chemical Coagulation and Electrocoagulation Process. PLOS ONE, v. 7, n. 6, p. e40108. doi:10.1371/journal.pone.0040108, 2012
BEHBAHANI, M.; MOGHADDAM, M. R. A.; ARAMI, M. (2011) Technoeconomical evaluation of fluoride removal by electrocoagulation process: optimization through response surface methodology. Desalination, v. 271, n. 1, p. 209-218.

BENSADOK, K.; BENAMMAR, S.; LAPICQUE, F.; NEZZAL, G. (2008) Electrocoagulation of cutting oil emulsions using aluminium plate electrodes. Journal of Hazardous Materials, v. 152, n. 1, p. 423-430.

BRASIL. Ministério do Meio Ambiente. Conselho Nacional do Meio Ambiente (Conama). (2011) Resolução n.o 430, de 13 de maio de 2011. Complementa e altera a Resolução n.0 357/2005. Dispõe sobre as condições e padrões de lançamento de efluentes, complementa e altera a Resolução n.o 357, de 17 de março de 2005, do Conselho Nacional do Meio Ambiente (Conama) Disponível em: <http://www.mma.gov.br/port/ conama/legiabre.cfm?codlegi=646>. Acesso em: 13 maio 2015.

BRASIL. Ministério do Meio Ambiente. Conselho Nacional do Meio Ambiente (Conama). (2002) Resolução n.o 313, de 29 de outubro de 2002. Revoga a Resolução Conama n. ${ }^{0}$ 6/88. Dispõe sobre o Inventário Nacional de Resíduos Sólidos Industriais. Disponível em: <http://www.mma.gov.br/port/conama/legislacao/CONAMA RES CONS_2002_313.pdf>. Acesso em: 10 abr. 2016.

CAÑIZARES, P.; CARMONA, M.; LOBATO, J.; MARTÍNEZ, F.; RODRIGO, M.A. (2005) Electrodissolution of aluminum electrodes in electrocoagulation processes. Industrial \& Engineering Chemistry Research, v. 44, n. 12, p. 4178-4185.

COMPANHIA DE SANEAMENTO BÁSICO DO ESTADO DE SÃO PAULO - Sabesp; CH2M HILL. (2002) Relatório de Estabelecimento de Diretrizes Técnicas, Econômicas e Institucionais e de Programa de Ação para Implementação de Sistema de Água de Reúso na RMSP. São Paulo. 
GARCÍA, A.G.; MIRANDA, V.M.; CIENFUEGOS, I.G.M.; SANCHEZ, P.T.A.; JUAREZ, M.C.; HERNANDEZ, I.L. (2015) Industrial wastewater treatment by electrocoagulation-electrooxidation processes powered by solar cells. Fuel, v. 149, p. 46-54.

GHOSH, D.; MEDHI, C.R.; PURKAIT, M.K. (2008) Treatment of fluoride containing drinking water by electrocoagulation using monopolar and bipolar electrode connections. Chemosphere, v. 73, n. 9, p. 1393-1400.

Hespanhol, I.; Mierzwa, J.C.; Rodrigues, L.D.B.; Silva, M.C.C. (2006) Manual de Conservação e Reúso de Água na Indústria. Rio de Janeiro: DIM. 28 p.

Kobya, M.; HIZ, H.; Senturk, E.; Aydiner, C.; Demirbas, E. (2006) Treatment of potato chips manufacturing wastewater by electrocoagulation. Desalination, v. 190, n. 1, p. 201-211.

KUMAR, M.F.; PONSELVAN, I.A.; MALVIYA, J.R.; SRIVASTAVA, V.C.; MALL, I.D. (2009) Treatment of bio-digester efluent by electrocoagulation using iron electrodes. Journal of Hazardous Materials, v. 165, n. 1-3, p. 345-352.

KURT, U.; GONULLU, M.T.; ILHAN, F.; VARINCA, K. (2008) Treatment of domestic wastewater by electrocoagulation in a cell with Fe-Fe electrodes. Environmental Engineering Science, v. 25, n. 2, p. 153-161.

KUSHWAHA, J.P.; SRIVASTAVA, V.C.; MALL, I.D. (2010) Organics removal from dairy waste water by electrochemical treatment and residue disposal. Separation and Purification Technology, v. 76, n. 2, p. 198-205.

LACASA, E.; CAÑIZARES, P.; SÁEZ, C.; MARTÍNEZ, F.; RODRIGO, M.A. (2013) Modelling and cost evaluation of electro-coagulation processes for the removal of anions from water. Separation and Purification Technology, v. 107, p. 219-227.

LEÃO, E.A.S: MATTA, M.A.S.; CAVALCANTE, I.N.; MARTINS, J.A.C:; DINIZ, C.G.; VASCONCELOS, Y.B.; CARMONA, K.M.; VANZIN, M.M. (2010) O reúSO da água: um estudo de caso na lavagem de veículos em lava-jato de Belém/ PA. In: XVI Congresso Brasileiro de Águas Subterrâneas e XVII Encontro Nacional de Perfuradores de Poços, São Luís, set. 2010. Anais.., 2010. Disponível em: <http://aguassubterraneas.abas.org/asubterraneas/\%20 article/download/22987/15104>. Acesso em: 18 set. 2013.

MAHMUT, B.; MURAT, E.; MEHMET, K. (2007) Treatment of the textile wastewater by electrocoagulation: economical evaluation. Chemical Engineering Journal, v. 128, n. 2-3, p. 155-161.

MANSOUR, L.B.; KESENTINI, I. (2008) Treatment of effluents from cardboard industry by coagulation-electroflotation. Journal of Hazardous Materials, v. 153, n. 3, p. 1.067-1.070.

MODIRSHAHLA, N.; BEHNAJADY, M.A.; KOOSHAIIAN, S. (2007) Investigation of the effect of different electrode connections on the removal efficiency of tartrazine from aqueous solutions by electrocoagulation. Dyes Pigments, v. 74, n. 2, p. 249-257.

MOHAMMAD, E.M.; MUTTUCUMARU, S. (2009) Fluoride removal by a continuous flow electrocoagulation reactor. Journal of Environmental Management, v. 90, n. 2, p. 1204-1212.

MUSQUERE, P.; ELLINGSEN, F.; VIK, E.A. (1983) Electrotechnics in drinking and waterwater. Water supply, 8 Special Subject, n. 2-3, p. 1-25.
PANIZZA, M.; CERISOLA, G. (2010a) Applicability of electrochemical methods to carwash wastewaters for reuse. Part 1: anodic oxidation with diamond and lead dioxide anodes. Journal of Electroanalytical Chemistry, v. 638, n. 1, p. 28-32.

PANIZZA, M.; CERISOLA, G. (2010b) Applicability of electrochemical methods to carwash wastewaters for reuse. Part 2: electrocoagulation and anodic oxidation integrated process. Journal of Electroanalytical Chemistry, v. 638, n. 2, p. 236-240.

PICHARD, T.; CATHLIFAUD, G.; MAZET, M.; VANDESTEENDAM, C. (2000) Cathodic dissolution in the electrocoagulation process using Al electrodes. Journal Environment Monitoring, v. 2, n. 1, p. 77-80.

PREFEITURA DO MUNICÍPIO DE SÃO PAULO. (2015) Lei n.o 16.160, de 13 de abril de 2015. Cria o Programa de Reúso de Água em postos de serviços e abastecimento de veículos e lava-rápidos no município de São Paulo, e dá outras providências. Disponível em: <http://www3. prefeitura.sp.gov.br/cadlem/secretarias/negocios juridicos/cadlem/ integra.asp?alt=14042015L\%20161600000>. Acesso em: 15 maio 2015.

SANTOS, E.G.; ALSINA, O.L.S.; SILVA, F.L.H. (2007) Desempenho de biomassas na adsorção de hidrocarbonetos leves em efluentes aquosos. Química Nova, v. 30, n. 2, p. 327-331.

SCOTTISH ENVIRONMENT PROTECTION AGENCY (SEPA). (2007) Disponivel em: https://www.gov.uk/government/uploads/system/ uploads/attachment_data/file/485190/pmho0307bmdx-e-e.pdf. Acesso em 14 jul. 2016.

TCHAMANGO, S.; NANSEU-NJIKI, C. P.; HADJIEV, E. N. D.; DARCHEN, A. (2010) Treatment of dairy effluents by electrocoagulation using aluminium electrodes. Science of the Total Environment, v. 408, n. 4. p. $947-952$.

THE TELEGRAPH. (2012) Plea to ban motorists washing their cars in the street. Disponível em: <http://www.telegraph.co.uk/motoring/ news/9550745/Plea-to-ban-motorists-washing-their-cars-in-thestreet.html>. Acesso em: 15 maio 2015

Tir, M.; Mostefa, N.M. (2008) Optimization of oil removal from oily wastewater by electrocoagulation using response surface method. Journal of Hazardous Materials, v. 158, n. 1, p. 107-115.

UNITED STATES ENVIRONMENTAL PROTECTION AGENCY (EPA). Sewage S/udge Incineration. Disponível em: https://www3.epa.gov/ ttnchie1/ap42/chO2/final/cO2sO2.pdf. Acesso em 11 jul. 2016

WANG, C-T.; CHOU, W-L.; KUO, Y-M. (2009) Removal of COD from laundry wastewater by electrocoagulation/electroflotation. Journal of Hazardous Materials, v. 164, n. 1, p. 81-86.

ZODI, S.; POTIER, O.; LAPICQUE, F.; LECLERC, J-P. (2009) Treatment of the textile wastewaters by electrocoagulation: effect of operating parameters on the sludge settling characteristics. Separation and Purification Technology, v. 69, n. 1, p. 29-36.

ZONGO, I.; MAIGA, A.H.; WÉTHÉ, L.; VALENTIN, G.; LECLERC, J.P.; PATERNOTTE, G.; LAPICQUE, F. (2009) Electrocoagulation for the treatment of textile wastewaters with $\mathrm{Al}$ or Fe electrodes: compared variations of COD levels, turbidity and absorbance. Journal of Hazardous Materials, v. 169, n. 1-3, p. 70-76. 